Paper

\title{
Screening for mechanical responses of proteins using coarse-grained elastic network models
}

\author{
Yuichi Togashi ${ }^{1,2,3 a)}$ \\ ${ }^{1}$ Research Center for the Mathematics on Chromatin Live Dynamics (RcMcD), \\ Hiroshima University \\ 1-3-1 Kagamiyama, Higashi-Hiroshima 739-8526, Japan \\ ${ }^{2}$ Department of Mathematical and Life Sciences, Graduate School of Science, \\ Hiroshima University \\ 1-3-1 Kagamiyama, Higashi-Hiroshima 739-8526, Japan \\ ${ }^{3}$ Applied Information Systems Research Division, Cybermedia Center, Osaka \\ University \\ 5-1 Mihogaoka, Ibaraki 567-0047, Japan \\ a) togashi@hiroshima-u.ac.jp
}

Received August 20, 2015; Revised November 18, 2015; Published April 1, 2016

\begin{abstract}
Owing to recent progress in structural biology, the structures of a number of proteins have been resolved and deposited into databases such as the Protein Data Bank (PDB). Many proteins function as molecular machines or show allosteric effects, which require integrated communication between different parts of the protein structure; however, the general principles underlying such communication are not yet clear. All-atom molecular dynamics simulation is a powerful tool for tracking molecular motion, but is still far too computationally costly to be applied widely. In this paper, we present a simple method for assessing the properties of mechanical communications using coarse-grained steered molecular dynamics simulations with elastic network models. The method was tested by screening for a certain mechanical property among protein structures in the PDB.
\end{abstract}

Key Words: protein structures, elastic network models, coarse-grained molecular dynamics, nonlinear mechanics, allostery, mechanical communication

\section{Introduction}

The cell contains a variety of proteins and protein complexes, many of which literally function as integrated machines. Proteins and complexes typically acquire free energy from reactions such as hydrolysis of ATP, and their functions can be regulated by interactions with other ligands. However, these catalytic, regulatory, and other active functions of proteins can also vary or be specific to different parts of the protein. Therefore, for the multiple components of a protein molecule or complex to work 
properly, information must be effectively transmitted between these parts.

Chemicals are often used as the main media for signal transduction between different molecules in the cell. By contrast, within a single molecule or complex, the information is likely to be transferred mechanically rather than chemically.

Molecular dynamics (MD) simulation is a powerful tool to study the mechanics of proteins, and is especially useful in combination with external forces, representing the so-called steered MD approach. This approach can be explained by analogy to experiments using optical tweezers or atomic force microscopes. In steered MD simulations, unlike experiments, any force can be applied to any atom to observe their motion; however, the computational cost of this procedure is huge. Indeed, use of all-atom models would make it currently impossible to exceed millisecond-level computation.

Hence, an approach is needed to coarse-grain and simplify this model. Typically, the first choice to achieve this goal is to approximate an amino acid residue as a coarse-grained element. Gō models are well-known examples of this class of coarse-grained model $[1,2]$. Elastic network models (ENMs) have become popular coarse-grained models because of their simplicity [3-11]. In an ENM, each amino acid residue is represented by a point-like particle, and the interaction between each pair of particles is approximated by a linear elastic spring.

Normal-mode analysis (NMA) is often adopted to analyze the behavior of particles in the ENM [12], and even publicly available as a service in the internet today [13-16]. Around the ground state, the motion is linearly approximated using a $3 N \times 3 N$ linearization matrix, where $N$ is the number of particles. By solving the eigenvalue problem of the matrix, we can obtain the normal modes. Note that this type of ENM is often referred to as an anisotropic network model (ANM) $[7,8]$. There is another class of ENMs, the so-called Gaussian network models (GNMs) [4,5], in which the anisotropic nature of interactions is omitted; instead, the normal modes of a GNM are obtained by solving the eigenvalue problem of the $N \times N$ connectivity matrix.

However, as we pointed out previously $[17,18]$, a common situation arises in which the overall deformation is large, and thus the motion is highly nonlinear. Nevertheless, the local strains are so small that the elastic description of interactions is still valid, which means that although the ENM is still valid, the NMA breaks down under such circumstances. For such cases, we can still run coarsegrained MD simulations by simply solving for the equation of motion numerically. Although exact comparison is difficult, the computational cost of this approach is likely several orders of magnitude lower than the all-atom MD.

Even though the model is crude, it can nonetheless reflect certain processes that cannot be observed in the realm of normal-mode analysis around the ground state, such as steric hindrance between domains. Indeed, we previously observed a sequence of motion in the conformational transition of the kinesin-like protein (KIF1A) motor [18]. Since the computational cost is low, we could even apply perturbations to hundreds of residues in a protein hundreds of times each in order to obtain a picture of the direction and strength of mechanical communication. For example, we have suggested the possible mechanical basis of the "strain-sensor" mechanism in myosin motors based on this approach [19].

In studies adopting this approach, including our own previous work, many simulation trials are used for one or several target proteins. In fact, because of the simplicity of the model, millions of trials are possible using even a small computer system, and can be applied to different types of proteins to extract general rules or to screen for a significant signature. Here, we propose a system to screen for a mechanical property among many structural datasets in the Protein Data Bank (PDB) [20]. We provide examples of mechanical communication profiles, and discuss the feasibility of applying the proposed screening system over the whole PDB database.

\section{Methods}

\subsection{Model}

As we apply forces to probe the mechanical transmission, the three-dimensional structure of a molecule is an important factor to consider. Therefore, as in our previous studies [17-19], we adopt an ANMtype elastic network model. Each amino acid residue in the protein is represented by a point-like particle. The positions of the $\alpha$-carbon $\left(\mathrm{C}^{\alpha}\right)$ atoms in the reference structure are used as the initial 
positions $\mathbf{R}_{i}^{(0)}(i=1, \cdots, N)$ of the particles, where $N$ is the number of particles. The forces acting between the particles are approximated as linear elastic springs. If the initial positions of two particles are within a certain cutoff distance, $l_{c}$, these particles are considered to be connected by a spring. The natural (equilibrium) length of the spring is set to be equal to the initial distance. All the springs are initially without strain, and hence, the reference conformation is the lowest internal energy state. Since the inter-residue interactions are introduced depending on the reference structure and the reference is set as the ground state, the model can be considered as a simplified form of the Gō-like models.

Our objective is not to precisely investigate a single protein but rather to sample or screen many structures. For the sake of simplicity, we set all the spring constants at the same values although the optimization of parameters is suggested; e.g., by fitting nuclear magnetic resonance data [21], which can be incorporated in the system if necessary. As we consider slow motion, we assume an overdamped limit, and hence, the system state is represented by the coordinates $\mathbf{R}_{i}(i=1, \cdots, N)$ of the particles. The viscous friction coefficient is set to be uniform for all particles. With the above assumptions, by the scaling of force and time, the spring constant and the viscous friction coefficient can be set to 1 without loss of generality. With the scaling, the force is measured in units of length i.e., $1 \AA$ force stretches a single spring by $1 \AA$.

The equation of motion is given by:

$$
\frac{d \mathbf{R}_{i}}{d t}=-\sum_{j=1}^{N} A_{i j} \frac{\mathbf{R}_{i}-\mathbf{R}_{j}}{\left|\mathbf{R}_{i}-\mathbf{R}_{j}\right|}\left(\left|\mathbf{R}_{i}-\mathbf{R}_{j}\right|-\left|\mathbf{R}_{i}^{(0)}-\mathbf{R}_{j}^{(0)}\right|\right)
$$

where $A_{i j}$ is the connectivity matrix; $A_{i j}=1$ if particles $i$ and $j$ are directly connected by a spring, and $A_{i j}=0$ otherwise. As mentioned above, the connectivity $A_{i j}$ is set according to the initial distance $d_{i j}^{(0)}=\left|\mathbf{R}_{i}^{(0)}-\mathbf{R}_{j}^{(0)}\right| ; A_{i j}=1$ for $d_{i j}^{(0)}<l_{c}$ and $A_{i j}=0$ otherwise. The cutoff distance $l_{c}$ is $10 \AA$.

\subsection{Structural datasets}

We adopted structural datasets deposited in the PDB, which provides yearly snapshots of the archive. We used the snapshot provided as of January 1, 2013, which contains 87,090 entries. For each file in the PDB format, "ATOM" records for $\mathrm{C}^{\alpha}$ were extracted, and their positions were listed. If there was more than one model for a chain or more than one $\mathrm{C}^{\alpha}$ position for a given residue, then we adopted the first record. According to the listed positions, an elastic network was constructed. The distribution of the numbers of amino acid residues detected is shown in Fig. 1. The average and root mean-squared numbers (excluding structures without amino acid residues) are 612 and 1035, respectively. These steps were automatized, and the time required to construct the networks was negligible compared to the time required for the simulation described below.

Table I shows the sample sets used in this study.

In the random sampling experiments, we randomly generated 4-character strings that conformed to the PDB ID rule, i.e., the first character is a number between 1 and 9 , and the latter three characters are either a number $(0-9)$ or letter $(\mathrm{A}-\mathrm{Z})$, and were used as PDB ID candidates (there were $9 \times 36^{3}=419,904$ possible IDs overall). We sampled 1,000 ID candidates and found 202 datasets in the archive, 197 of which contained proteins (Sample RS). These 197 samples were used, ignoring other associated molecules, such as ligands or nucleic acids. The numbers of residues therein range from 24 to 3,670. The average and root mean-squared numbers are 608 and 873 , respectively, which are close to those of all the datasets in the snapshot. Hence, we adopted these samples to estimate the computational costs for a survey over the entire PDB.

In the screening experiments over a certain size range, we used all of the PDB files that contained a certain range of numbers of residues $\left(\mathrm{C}^{\alpha}\right)$. We targeted relatively large protein structures, and set three ranges: 1000-1004 (Sample 1k; 73 datasets), 2000-2019 (Sample 2k; 72 datasets), and 5000-5199 (Sample 5k; 36 datasets) residues. 


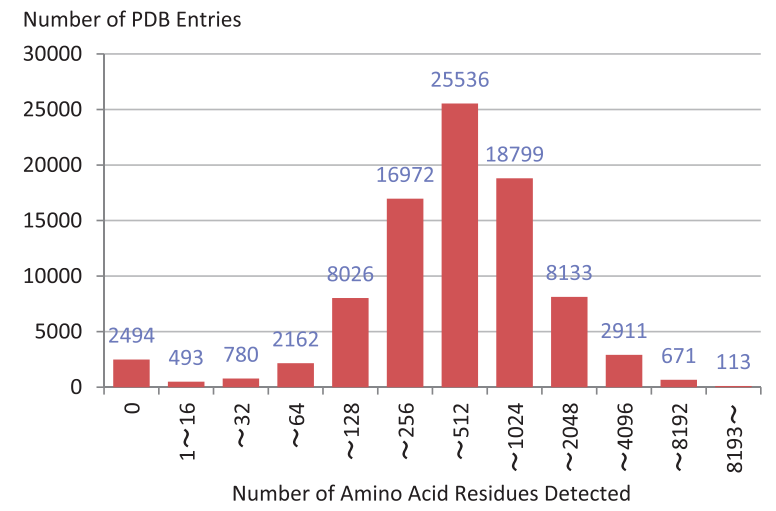

Fig. 1. Distribution of the numbers of amino acid residues detected in each PDB entry.

Table I. List of sample datasets.

\begin{tabular}{l|c|c|l}
\hline Name & $\begin{array}{c}\text { Number of } \\
\text { datasets }\end{array}$ & $\begin{array}{c}\text { Range of the size } \\
\text { (number of residues) }\end{array}$ & Description \\
\hline Sample RS & 197 & $24-3670$ & Randomly sampled from the PDB. \\
\hline Sample 1k & 73 & $1000-1004$ & All PDB files within the size range shown. \\
Sample 2k & 72 & $2000-2019$ & All PDB files within the size range shown. \\
Sample 5k & 36 & $5000-5199$ & All PDB files within the size range shown. \\
\hline
\end{tabular}

\subsection{Force and trap}

To study the mechanical response, we use forces on certain particles in the elastic network. One method is to put attractive or repulsive forces between pairs of particles. Such a pair of forces can be introduced either by constant strength of force towards or away from the other particle, by addition of a spring whose equilibrium distance is shorter or longer than the initial distance, or by any other form e.g. Lennard-Jones potential. Such pairwise central forces within the molecule do not induce global translation or rotation motion, and hence, it is not needed to prevent the molecule from drifting.

It is analogous to change of chemical property of the residue, or binding of a ligand there. To represent ligand binding, it is also possible to introduce a virtual ligand represented by additional particles and consider forces between the protein and ligand. Similar attempts have been made by steered or guided MD simulations [17,22,23].

In this work, we simply use a constant strength of attractive forces between the target particle (force application point) and all the particles directly connected to the target by a spring (Case A). It is equivalent to the decrease of the equilibrium length of the spring. The overall equation of motion is given by

$$
\frac{d \mathbf{R}_{i}}{d t}=-\sum_{j=1}^{N} A_{i j} \frac{\mathbf{R}_{i}-\mathbf{R}_{j}}{\left|\mathbf{R}_{i}-\mathbf{R}_{j}\right|}\left(\left|\mathbf{R}_{i}-\mathbf{R}_{j}\right|-\left|\mathbf{R}_{i}^{(0)}-\mathbf{R}_{j}^{(0)}\right|+f_{e x t, i j}\right),
$$

where, $f_{\text {ext,ij }}$ is the strength of the force between particles $i$ and $j$, which is nonzero only if either $i$ or $j$ is the force application point.

Another method is to probe by using single, point forces. It may correspond to the external forces induced by other parts of the molecular complex (e.g., the other motor domain in a double-headed motor [19]), or force-spectroscopy experiments (e.g. stretching) using atomic force microscopy. Here, we apply a static force to a single residue in each simulation trial.

Unlike the pairwise forces, such point forces generally induce global translation and rotation of the molecule, which is inconvenient for investigation of mechanical properties inside the molecule. Hence, we need to trap the molecule.

We can mathematically cancel the global translation and rotation. Again, we use the same method as in our previous work [19] (Case B). We consider such a coordinate frame that the origin is the center of mass of the molecule. Assuming that a force $\mathbf{F}_{\text {ext }}$ is applied to node $a$, then, the trapping force on node $i$ to cancel the global translation and rotation is 


$$
\mathbf{f}_{i}=-\frac{\mathbf{F}_{e x t}}{N}-\left[\mathrm{M}^{-1}\left(\mathbf{R}_{a} \times \mathbf{F}_{e x t}\right)\right] \times \mathbf{R}_{i}
$$

where

$$
\mathrm{M}=\sum_{i=1}^{N}\left(\begin{array}{ccc}
\left|\mathbf{R}_{i}\right|^{2}-X_{i}^{2} & -X_{i} Y_{i} & -X_{i} Z_{i} \\
-X_{i} Y_{i} & \left|\mathbf{R}_{i}\right|^{2}-Y_{i}^{2} & -Y_{i} Z_{i} \\
-X_{i} Z_{i} & -Y_{i} Z_{i} & \left|\mathbf{R}_{i}\right|^{2}-Z_{i}^{2}
\end{array}\right)
$$

$\left(\mathbf{R}_{i}={ }^{t}\left(X_{i}, Y_{i}, Z_{i}\right)\right.$; see [19] for derivation). By adding $\mathbf{f}_{i}$ (and also $\mathbf{F}_{e x t}$ for node $a$ ) to the right hand side of Eq. (1), the molecule can be trapped. This method has an advantage that it does not induce any internal strains.

Another possibility is to weakly anchor the particles in the model around their original positions. Here, all the particles except the force application point are harmonically trapped at their original positions (Case $\mathrm{C}$ ). The overall equation of motion is

$$
\frac{d \mathbf{R}_{i}}{d t}=\mathbf{F}_{e x t, i}-k_{t r a p, i}\left(\mathbf{R}_{i}-\mathbf{R}_{i}^{(0)}\right)-\sum_{j=1}^{N} A_{i j} \frac{\mathbf{R}_{i}-\mathbf{R}_{j}}{\left|\mathbf{R}_{i}-\mathbf{R}_{j}\right|}\left(\left|\mathbf{R}_{i}-\mathbf{R}_{j}\right|-\left|\mathbf{R}_{i}^{(0)}-\mathbf{R}_{j}^{(0)}\right|\right)
$$

with the static force $\mathbf{F}_{e x t, i}$, which is nonzero only for the designated residue (i.e., the force application point) $i$. The spring constant $k_{t r a p, i}$ for the trap is set to 0.01 , which is soft enough to allow for deformations by probing forces, except for the force application point where $k_{t r a p, i}=0$ (Note: with the scaling introduced above, the force is given in units of length, and thus the spring constant is dimensionless; the spring constant 1 corresponds to that of a single spring in the elastic network).

These cases are summarized in Table II. In Case A (pairwise forces), the strength of the force was set to $(1 / \sqrt{n}) \AA$, where $n$ is the number of forced pairs, i.e., the number of springs directly connected to the force application point. In Cases B and C (point force), the strength of the force was set to 1 $\AA$, and the direction was randomly chosen.

Table II. Comparison of the perturbation and trapping methods.

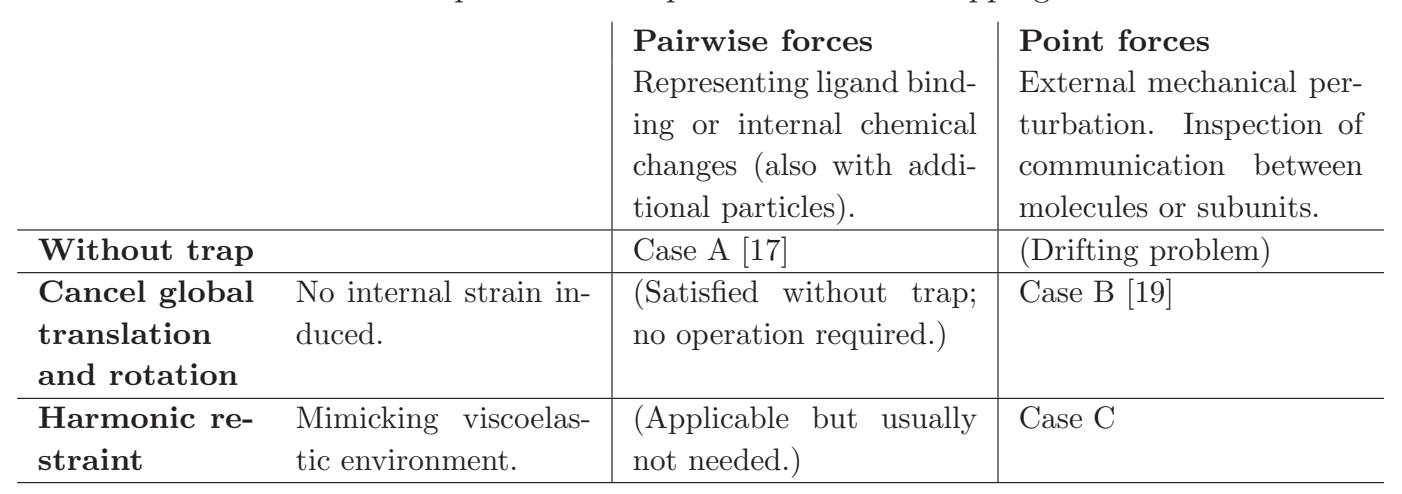

\subsection{Simulation and analysis}

We numerically simulated the dynamics of the ENM under the forces represented by the above equation of motion. The integration was carried out by the 4th-order Runge-Kutta method, with a time step of 0.1 .

After a designated time span that is long enough to allow conformational relaxation, we can obtain the positions of residues, and calculate the changes from the initial (reference) conformation. With a point force, the changes generally depend on the direction of the static force applied. Typically, the simulation trials are repeated for the same force application point to probe the direction that induces a strong response.

To analyze the structural response, we can define different types of measures depending on the purpose. For example, if our interest is mainly on domain motion, changes in pairwise distances between residues belonging to different domains may reflect such motion. An example is shown in 
Fig. 2. An $N \times N$ matrix of distance changes is obtained for each force application point, and the domain structures can be observed.

However, the actual number of variables (degrees of freedom) is only $3 N$. Such matrices are redundant and not very convenient for automated screening. In our previous work [19], we chose some residues as markers, and showed the changes in distances between them. However, the choice of markers depends on prior information, such as known functional domains, although there are preceding studies to determine domains from such distance changes [22].

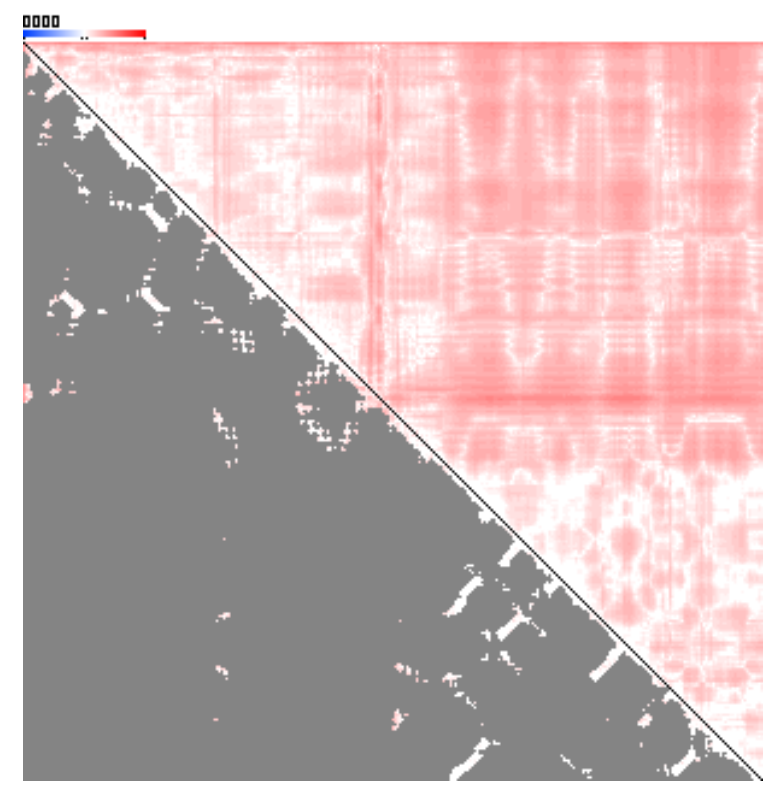

Fig. 2. Distance changes between residues: case of phosphatase and tensin homolog (PTEN). A total of 307 residues from the structural dataset (PDB ID: 1D5R [24]) are displayed from the top left (N-terminal) to the bottom right (Cterminal), and the distance changes between them are shown in different colors. In each trial, the static force (strength $1 \AA$ ) at a randomly chosen direction is applied to the N-terminal residue Arg14 (Case C). The waiting time is 10,000 time units. The maximum value of the absolute distance change in 100 trials is calculated for each pair of residues; darker red indicates larger changes. In the bottom-left half, residue pairs that are not directly connected are grayed out; i.e., the color indicates the strain of the spring between the residues. This protein consists of two subdomains, and distance changes between residues in different subdomains (top-right square) are relatively large, reflecting the repositioning between stiff subdomains.

Here, we use two simple measures for screening: the root mean-squared displacement (RMSD) of each particle and the root mean-squared strain (RMSS) of each spring. The RMSD shows how conformational motions are transmitted, and the RMSS represents how internal strains propagate over the molecule. These are generally not proportional. For example, if strains are concentrated around a hinge-like structure, the particles away from the hinge can show large motion without strains in their neighboring springs.

The effect of the external force depends on the distance from the force application point. In this work, we surveyed the RMSD and RMSS for each 1- $\AA$ bin of the distance (for RMSS, distance to the nearer end of the spring was used).

\section{Results}

\subsection{Mechanical communication patterns}

As stated in the previous section, we applied structural perturbation by using external forces and measured the RMSD and RMSS. Figure 3 shows the case of Sample RS (197 randomly chosen samples). The force application point itself shows a wide range of displacements. Hence, instead of using the raw RMSD values, we normalized the RMSD to that of the force application point, as shown 

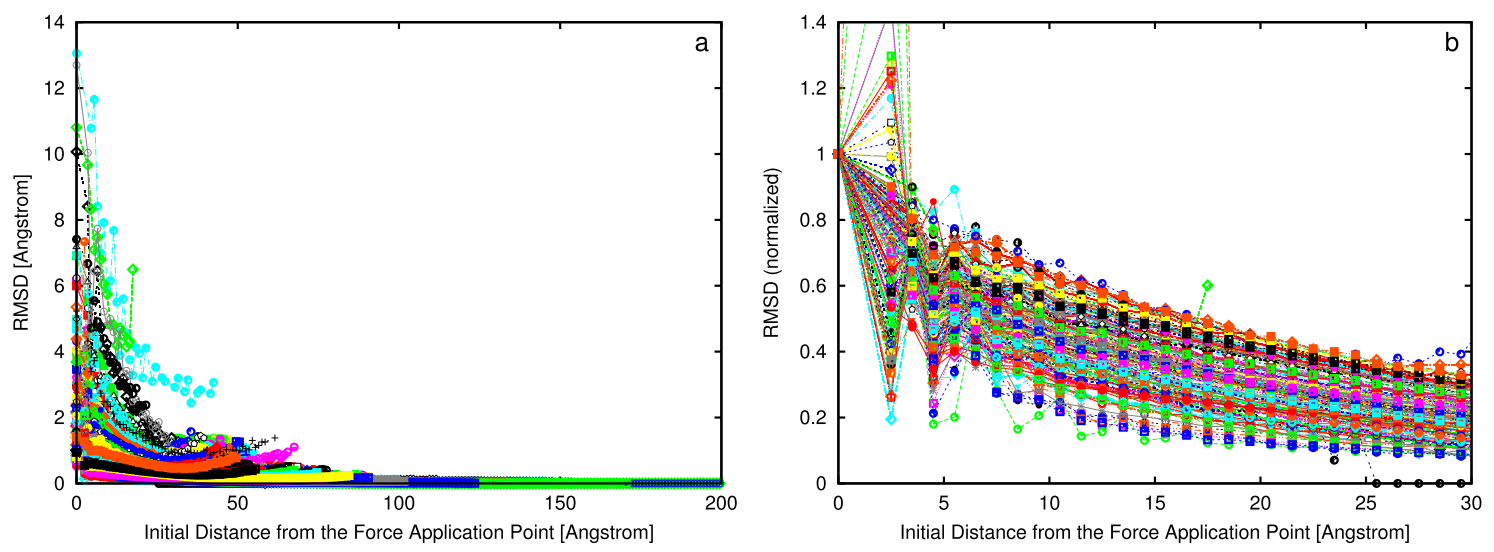

Fig. 3. Root mean-squared displacements, plotted against the initial distance from the force application point. (a) Raw RMSD values, and (b) RMSD values normalized to the displacement of the force application point. Case C, Sample RS: 197 randomly chosen samples were used; each line corresponds to a sample dataset. The waiting time is 2,000 time units; 1 trial for each force application point (these conditions for the simulations are also applied to Figs. 4 to 7).

in Fig. 3(b). The RMSD largely decayed with the distance from the force application point; however, the neighboring residues sometimes moved even more than the force application point. The RMSS is also normalized in the same way to that of the spring directly connected to the force application point (zero distance).

To compare mechanical properties among proteins, we next screened similar-sized structures. Figure 4 shows the result for Samples $1 \mathrm{k}$ and $2 \mathrm{k}$ (with roughly 1,000 or 2,000 residues). The normalized RMSD showed large variation at long distances, which is convenient as a simple criterion of screening. In larger structures (Sample 5k), as shown in Fig. 5, the RMSD largely decayed exponentially with increasing distance, but sometimes showed stepwise drops, reflecting domain boundaries.

The result also depends on the forcing and trapping methods. Although these cases correspond to different situations and the best choice may vary, comparison between them would be informative. Figure 6 shows the RMSD and RMSS for Sample RS, in Cases A, B, and C. As seen in Fig. 6(a), the mathematical trap (Case B) often induces artifacts in the RMSD due to the cancellation of global rotation, and the harmonic restraint (Case $\mathrm{C}$ ) for small molecules may also cause rotation issue. These methods work well for the RMSS; especially, the mathematical trap does not induce extra internal strains.

We plotted several pairs of measures in Fig. 7. Basically, the correlation between the RMSD and RMSS in each case is generally not significant, and the correlation of the RMSD or RMSS between different cases is also poor. However, the RMSD in Case A (pairwise forces) and the RMSS in Case B (point forces) showed strong correlation. It implies, even though we probed each residue only once in these cases, the relationship between the applied strain on the springs and resulting motion of particles is already in good correlation with that between the applied displacement and resulting strains.

\subsection{Computational costs}

For effective screening over a large number of datasets, reduction of computational costs is crucial. The ENM is a very light-weight model, in which only a limited number and simple form of interactions are considered.

For the random sampling experiments (Sample RS; 197 datasets), we used 64 simulation threads running on a PC server with $64 \mathrm{CPU}$ cores (four AMD Opteron 6282SE running at $2.6 \mathrm{GHz} ; 64 \mathrm{~GB}$ memory; linux 2.6.32, icc 14.0.3). The total elapsed time for the simulation was 14.0, 15.3, and 14.3 $\mathrm{h}$ for Case A, B, and C, respectively (median in 3 trials each); corresponding to ca. 310-340 datasets per day. Although we probed each residue only once each in this case, this result shows that it is feasible to conduct this type of simple survey over the whole PDB using a small PC cluster in a reasonable time period (within months). 

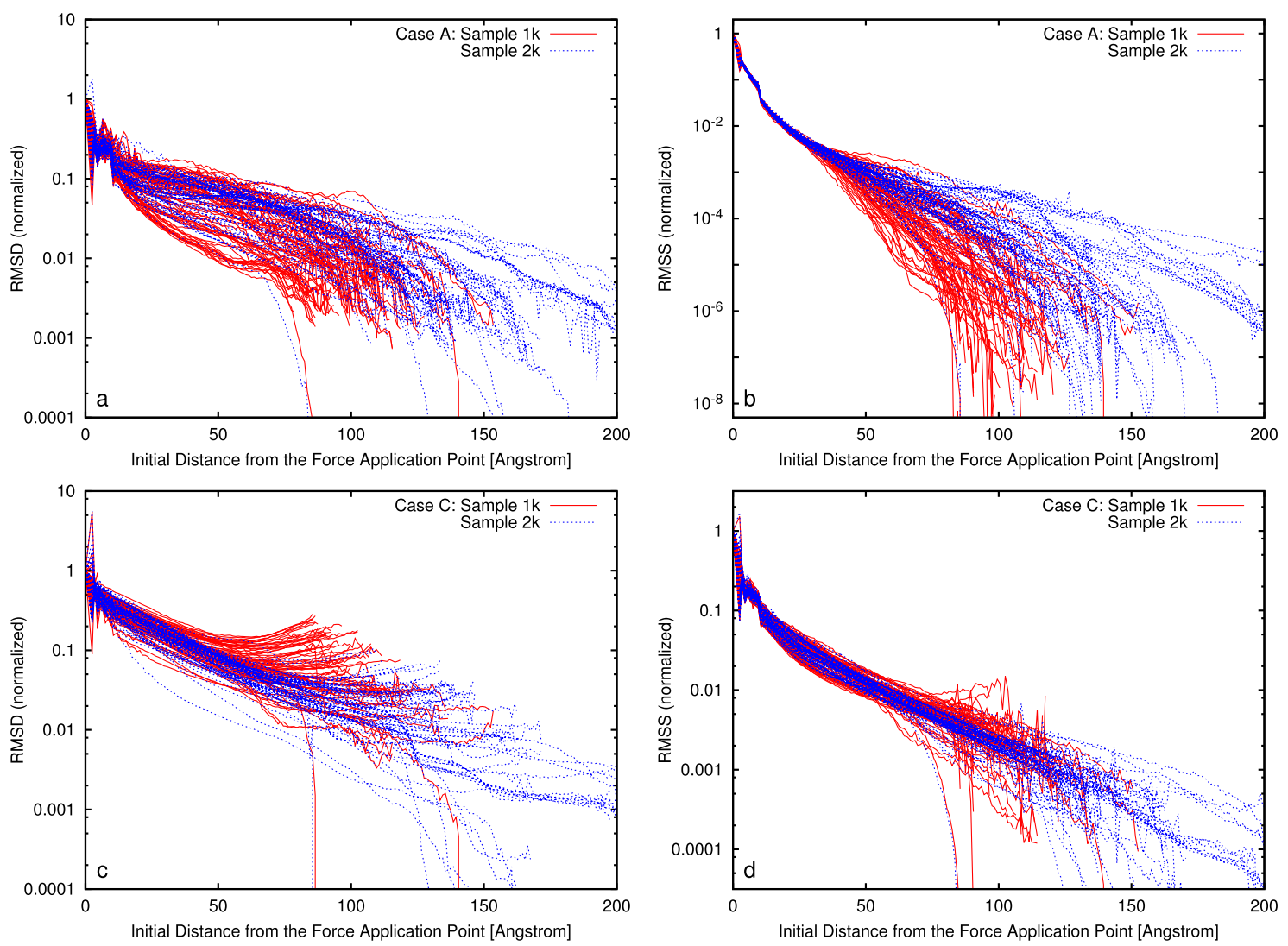

Fig. 4. Normalized RMSD (a, c) and RMSS (b, d), plotted against the initial distance from the force application point. Case A (a, b) and Case C (c, d). Samples $1 \mathrm{k}$ (red) and 2k (blue) were used; each line corresponds to a given dataset.

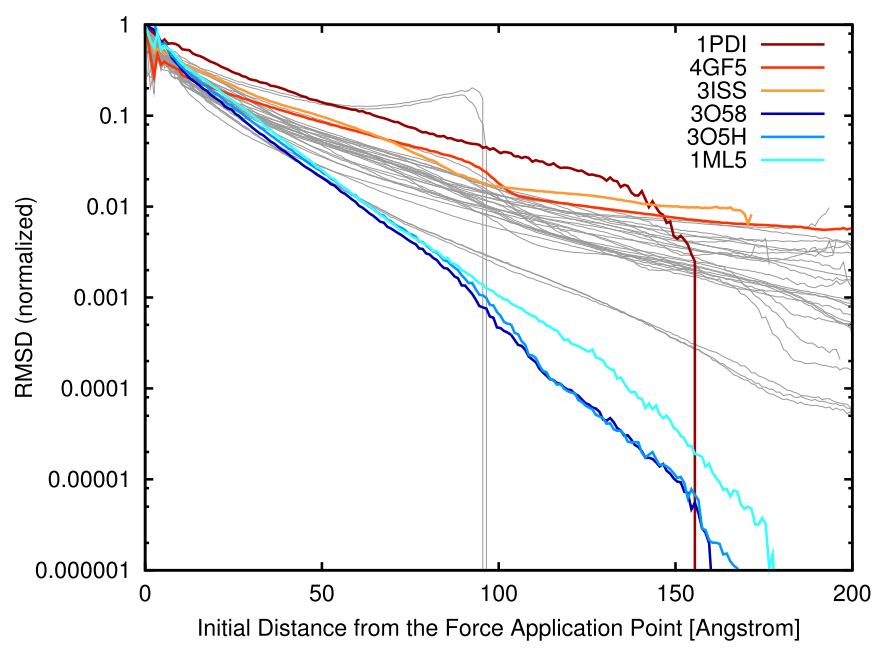

Fig. 5. Normalized RMSD in Case C, plotted against the initial distance from the force application point. Sample $5 \mathrm{k}$ was used, and the results are shown in the same way as described in Fig. 4. The colored lines correspond to the structures with the PDB IDs indicated. 

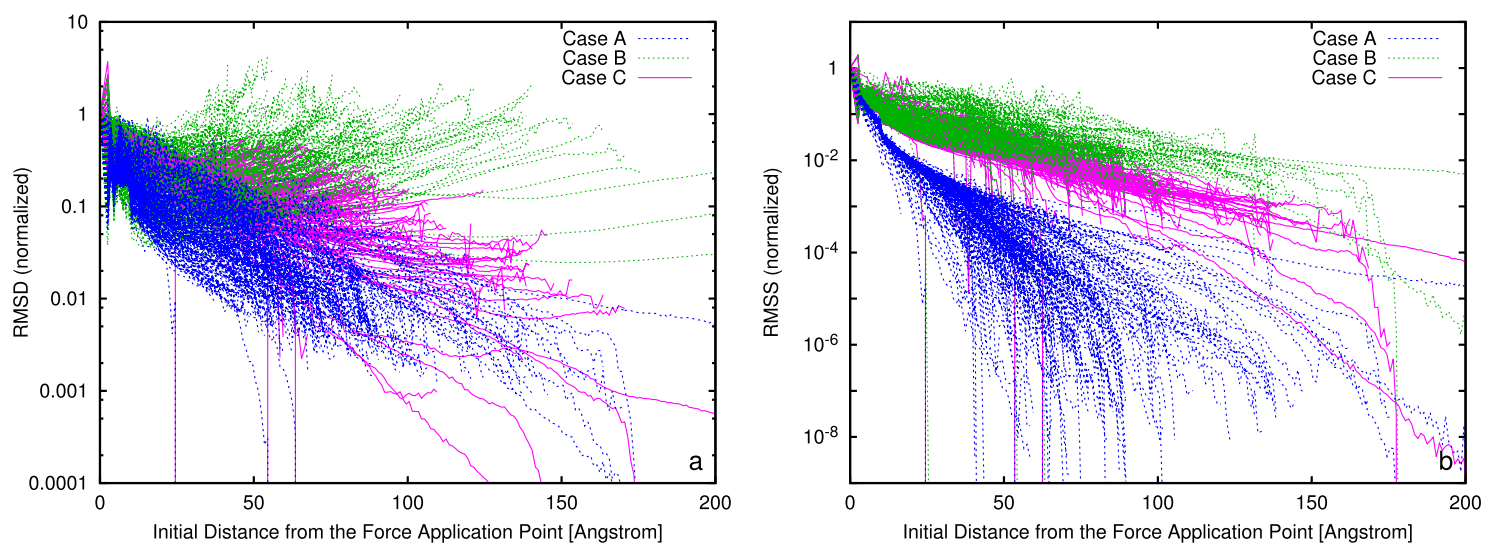

Fig. 6. Comparison of forcing and trapping methods. Sample RS; (a) normalized RMSD and (b) normalized RMSS in (blue) Case A, (green) Case B, and (purple) Case C, plotted against the initial distance from the force application point.
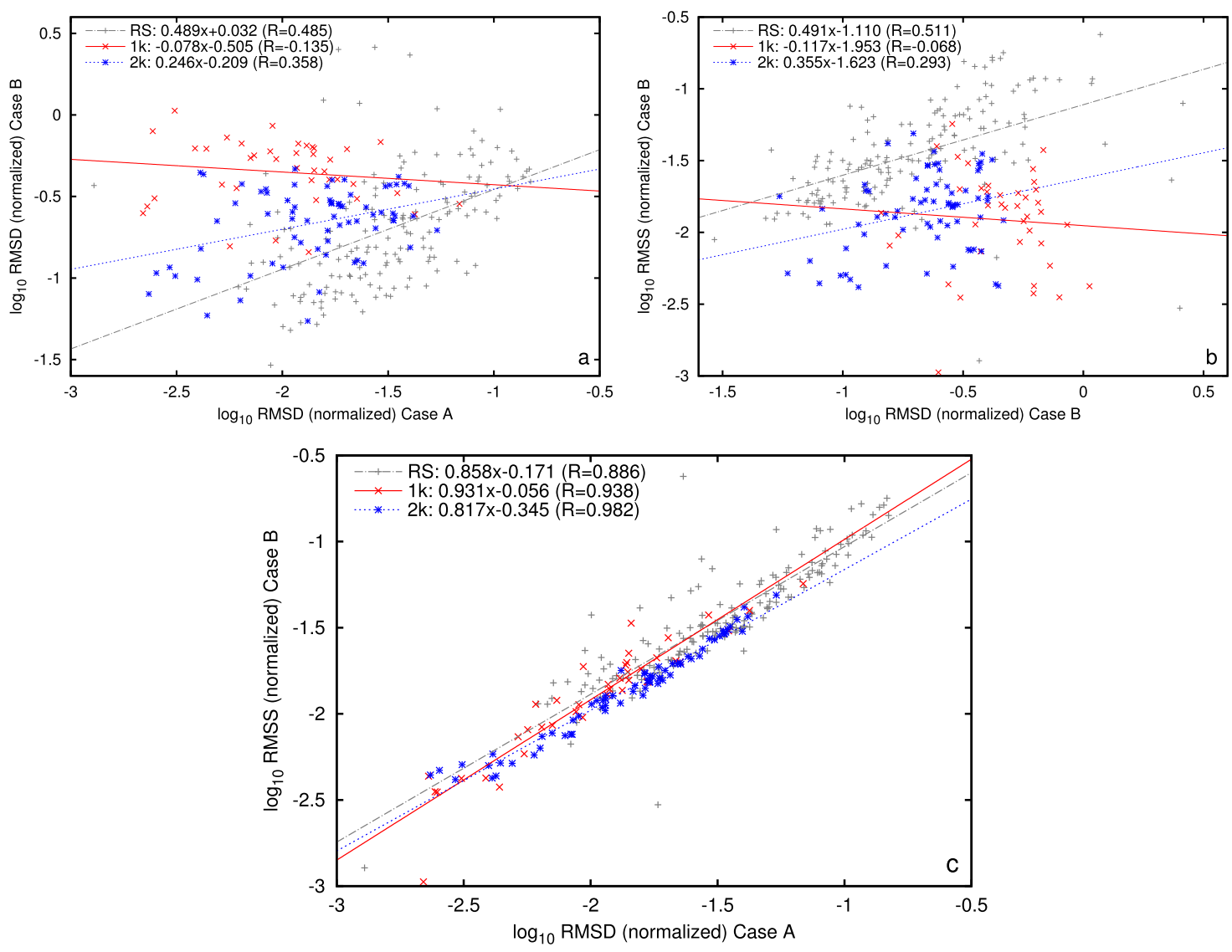

Fig. 7. Correlation between different cases and between the RMSD and RMSS (normalized). (a) RMSD in Case A and B, (b) RMSD and RMSS in Case B, and (c) RMSD in Case A and RMSS in Case B. Only samples in which both RMSD and RMSS are nonzero values (i.e., motion and strains are transmitted over the distance) were used; (gray) Sample RS (164 out of 197), at a distance of 50-51 $\AA$, (red) Sample $1 \mathrm{k}$ (40 out of 73 ), at a distance of 100-101 $\AA$, and (blue) Sample 2k (71 out of 72), at a distance of 100-101. Linear regression was applied after taking logarithm; correlation coefficient $R$ is shown for each case. 
The equation of motion is sometimes very stiff (showing a wide range of normal-mode frequencies), and thus, implicit algorithms may be useful to accelerate the final stage of convergence. When considering only the final state, simpler convergent (root-finding) algorithms may be used.

\section{Discussion}

The main objective of this study was to find a suitable and computationally light approach to screen for proteins with a certain mechanical property. To show the feasibility of this approach, we chose structural datasets at random or all the data within a certain size range. The computational cost was low enough to allow for screening over the entire database, at least with the simple criteria adopted herein.

\subsection{General properties of communication}

For large proteins, the residue displacements generally decay exponentially with increasing distance from the force application point, as shown in Fig. 5. However, the decay rates vary, reflecting the mechanical properties of the molecule. In the same way, we can determine variation in the stiffness among different subunits.

Some irregular datasets may have affected the results of the present analysis. In particular, the structures showing the weakest levels of communication sampled here (PDB IDs: $3 \mathrm{O} 58$ and 3O5H) are partial datasets including nucleic acids. Although such irregular data will need to be excluded manually in our current system using the PDB format, PDB initiated the use of new formats of protein structures, such as PDBx/mmCIF and PDBML, as their standard in 2014. These improved formats can at least help to eliminate problems with such partial (divided) data, and will also be suitable for automated processing.

Analyses based only on the networks (adjacency matrices) while ignoring the original threedimensional positions have been conducted by many other investigators, and have been shown to be informative [25-27]. Comparison with such works may also help to elucidate the general applicability of GNM-type models.

\subsection{Automated screening and its applications}

The mechanical responses of ENMs to external forces have been discussed based on NMA [28]. However, as pointed out in previous papers $[17,18]$, the nonlinear nature of dynamics strongly affects the motion. In the context of communication, such nonlinearity may cause the communication to be almost one directional, i.e., the force to domain A can deform domain B but not vice versa. Such nonlinear effects cannot be studied in the realm of normal-mode analysis, and are thus good targets of our proposed screening method.

The behavior of ENMs can be compared with experiments, e.g., single molecule force spectroscopy [29]. A shortcoming of ENM-based methods is that ENMs cannot reproduce unfolding. Partial unfolding is involved in the operation of some protein machines. Although Gō-like models are often the first choice for such systems, and indeed used for a mechanical stretching survey of thousands of proteins [30], variants of ENMs can be also considered to reduce computational costs [31-34].

In previous papers, the binding of ATP is mimicked by adding attractive forces between residues in the nucleotide-binding pocket and some fictitious particle(s) representing ATP $[17,35,36]$. Such forces applied to multiple residues can be used with the proposed approach. Pairwise attractive or repulsive forces are convenient, as they do not induce total translation and rotation, and can be used as a model of ligand binding at a certain site of a protein.

Beyond single proteins, ENM-like models have also been recently employed to analyses of large polymers and complexes [37]. Such large objects (e.g., chromosomes) show dynamic motion in the cell, which may be related to their function. However, little is known about the underlying mechanisms contributing to such motion. Our mechanical screening method can be combined with artificial structures and evolutionary simulations in order to extract valuable information on the general relationship among molecular structures, motion, and functions $[17,38]$. 


\section{Acknowledgments}

The author is grateful to Shoji Takada, Alexander S. Mikhailov, Hiroaki Takagi, Shin-ichi Tate, and Holger Flechsig for their helpful comments. This work was supported by the Ministry of Education, Culture, Sports, Science, and Technology, Japan (KAKENHI 23115007 "Spying minority in biological phenomena," and Platform for Dynamic Approaches to Living System) and the Japan Agency for Medical Research and Development. The large-scale computing system of the Cybermedia Center, Osaka University was used for part of this study.

\section{References}

[1] N. Gō, "Theoretical studies of protein folding," Ann. Rev. Biophys. Bioeng., vol. 12, pp. 183210, June 1983.

[2] S. Takada, "Gō-ing for the prediction of protein folding mechanisms," Proc. Natl. Acad. Sci. (USA), vol. 96, no. 21, pp. 11698-11700, October 1999.

[3] M.M. Tirion, "Large amplitude elastic motions in proteins from a single-parameter, atomic analysis," Phys. Rev. Lett., vol. 77, no. 9, pp. 1905-1908, April 1996.

[4] I. Bahar, A.R. Atilgan, and B. Erman, "Direct evaluation of thermal fluctuations in proteins using a single-parameter harmonic potential," Folding. Des., vol. 2, no. 3, pp. 173-181, May 1997.

[5] T. Haliloglu, I. Bahar, and B. Erman, "Gaussian dynamics of folded proteins," Phys. Rev. Lett., vol. 79, no. 16, pp. 3090-3093, April 1997.

[6] K. Hinsen, "Analysis of domain motions by approximate normal mode calculations," Proteins, vol. 33, no. 3, pp. 417-429, November 1998.

[7] P. Doruker, A.R. Atilgan, and I. Bahar, "Dynamics of proteins predicted by molecular dynamics simulations and analytical approaches: application to $\alpha$-amylase inhibitor," Proteins, vol. 40, no. 3, pp. 512-524, August 2000.

[8] A.R. Atilgan, S.R. Durell, R.L. Jernigan, M.C. Demirel, O. Keskin, and I. Bahar, "Anisotropy of fluctuation dynamics of proteins with an elastic network model," Biophys. J., vol. 80, no. 1, pp. 505-515, January 2001.

[9] F. Tama and Y.-H. Sanejouand, "Conformational change of proteins arising from normal mode calculations," Protein Eng., vol. 14, no. 1, pp. 1-6, January 2001.

[10] E. Eyal, A. Dutta, and I. Bahar, "Cooperative dynamics of proteins unraveled by network models," WIREs Comput. Mol. Sci., vol. 1, no. 3, pp. 426-439, May 2011.

[11] I. Bahar, M.H. Cheng, J.Y. Lee, C. Kaya, and S. Zhang, "Structure-encoded global motions and their role in mediating protein-substrate interactions," Biophys. J., vol. 109, no. 6, pp. 11011109, September 2015.

[12] Ed. Q. Cui and I. Bahar, Normal Mode Analysis: Theory and Applications to Biological and Chemical Systems, Chapman \& Hall/CRC, Boca Raton, 2006.

[13] Anisotropic Network Model Web Server 2.0 http://www.ccbb.pitt.edu/anm/.

[14] E. Eyal, G. Lum, and I. Bahar, "The anisotropic network model web server at 2015 (ANM 2.0)," Bioinformatics, vol. 31, no. 9, pp. 1487-1489, January 2015.

[15] PDBj ProMode Elastic http://promode.pdbj.org/promode_elastic/.

[16] H. Wako and S. Endo, "Normal mode analysis based on an elastic network model for biomolecules in the Protein Data Bank, which uses dihedral angles as independent variables," Comp. Biol. Chem., vol. 44, pp. 22-30, June 2013.

[17] Y. Togashi and A.S. Mikhailov, "Nonlinear relaxation dynamics in elastic networks and design principles of molecular machines," Proc. Natl. Acad. Sci. (USA), vol. 104, no. 21, pp. 8697-8702, May 2007.

[18] Y. Togashi, T. Yanagida, and A.S. Mikhailov, "Nonlinearity of mechanochemical motions in motor proteins," PLoS Comput. Biol., vol. 6, no. 6, e1000814, June 2010.

[19] M. Düttmann, Y. Togashi, T. Yanagida, and A.S. Mikhailov, "Myosin-V as a mechanical sensor: an elastic network study," Biophys. J., vol. 102, no. 3, pp. 542-551, February 2012. 
[20] The Worldwide Protein Data Bank http://www.wwpdb.org/.

[21] Y. Dehouck and A.S. Mikhailov, "Effective harmonic potentials: insights into the internal cooperativity and sequence-specificity of protein dynamics," PLoS Comput. Biol., vol. 9, no. 8, e1003209, August 2013.

[22] I. Navizet, F. Cailliez, and R. Lavery, "Probing protein mechanics: residue-level properties and their use in defining domains," Biophys. J., vol. 87, no. 3, pp. 1426-1435, September 2004.

[23] M. Ikeguchi, J. Ueno, M. Sato, and A. Kidera, "Protein structural change upon ligand binding: linear response theory," Phys. Rev. Lett., vol. 94, no. 7, 078102, February 2005.

[24] J.-O. Lee, H. Yang, M.-M. Georgescu, A. Di Cristofano, T. Maehama, Y. Shi, J.E. Dixon, P. Pandolfi, and N.P. Pavletich, "Crystal structure of the PTEN tumor suppressor: implications for its phosphoinositide phosphatase activity and membrane association," Cell, vol. 99, no. 3, pp. 323-334, October 1999.

[25] H. Morita and M. Takano, "Residue network in protein native structure belongs to the universality class of a three-dimensional critical percolation cluster," Phys. Rev. E, vol. 79, no. 2, 020901, February 2009.

[26] L. Di Paola, M. De Ruvo, P. Paci, D. Santoni, and A. Giuliani, "Protein contact networks: an emerging paradigm in chemistry," Chem. Rev., vol. 113, no. 3, pp. 1598-1613, November 2012.

[27] W. Yan, J. Zhou, M. Sun, J. Chen, G. Hu, and B. Shen, "The construction of an amino acid network for understanding protein structure and function," Amino Acids, vol. 46, no. 6, pp. 1419-1439, March 2014.

[28] E. Eyal and I. Bahar, "Toward a molecular understanding of the anisotropic response of proteins to external forces: insights from elastic network models," Biophys. J., vol. 94, no. 9, pp. 34243435, May 2008.

[29] H. Dietz and M. Rief, "Elastic bond network model for protein unfolding mechanics," Phys. Rev. Lett., vol. 100, no. 9, 098101, March 2008.

[30] J.I. Sułkowska and M. Cieplak, "Stretching to understand proteins - a survey of the Protein Data Bank," Biophys. J., vol. 94, no. 1, pp. 6-13, January 2008.

[31] J. Higo and H. Umeyama, "Protein dynamics determined by backbone conformation and atom packing," Protein Eng., vol. 10, no. 4, pp. 373-380, April 1997.

[32] M.K. Kim, G.S. Chirikjian, and R.L. Jernigan, "Elastic models of conformational transitions in macromolecules," J. Mol. Graph. Model., vol. 21, no. 2, pp. 151-160, October 2002.

[33] O. Miyashita, J.N. Onuchic, and P.G. Wolynes, "Nonlinear elasticity, proteinquakes, and the energy landscapes of functional transitions in proteins," Proc. Natl. Acad. Sci. (USA), vol. 100, no. 22, pp. 12570-12575, October 2003.

[34] P. Maragakis and M. Karplus, "Large amplitude conformational change in proteins explored with a plastic network model: adenylate kinase," J. Mol. Biol., vol. 352, no. 4, pp. 807-822, September 2005.

[35] M. Düttmann, M. Mittnenzweig, Y. Togashi, T. Yanagida, and A.S. Mikhailov, "Complex intramolecular mechanics of G-actin - an elastic network study," PLoS ONE, vol. 7, no. 10, e45859, October 2012.

[36] H. Flechsig and A.S. Mikhailov, "Tracing entire operation cycles of molecular motor hepatitis C virus helicase in structurally resolved dynamical simulations," Proc. Natl. Acad. Sci. (USA), vol. 107, no. 49, pp. 20875-20880, December 2010.

[37] V.A. Avetisov, V.A. Ivanov, D.A. Meshkov, and S.K. Nechaev, "Fractal globules: a new approach to artificial molecular machines," Biophys. J., vol. 107, no. 10, pp. 2361-2368, November 2014.

[38] J.D. Fernández and F. Vico, "Automating the search of molecular motor templates by evolutionary methods," BioSystems, vol. 106, no. 2-3, pp. 82-93, November 2011. 